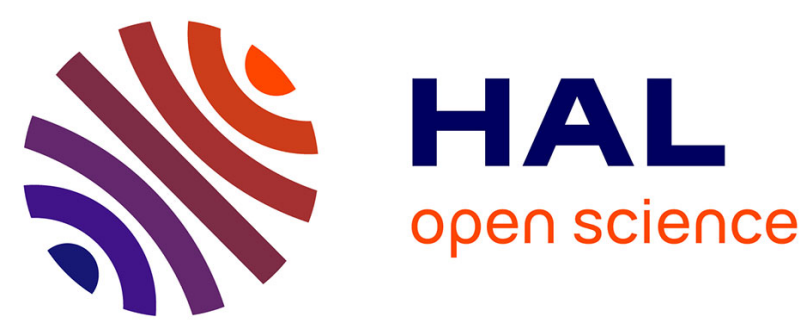

\title{
Exercises in Unstyling Texts: Formalisation and Visualisation of a Narrative's [Space, Time, Actors, Motion] Components
}

Jean-Yves Blaise, Iwona Dudek

\section{- To cite this version:}

Jean-Yves Blaise, Iwona Dudek. Exercises in Unstyling Texts: Formalisation and Visualisation of a Narrative's [Space, Time, Actors, Motion] Components. Knowledge Discovery, Knowledge Engineering and Knowledge Management, 914, pp.28-53, 2018, Communications in Computer and Information Science, 978-3-319-99700-1. 10.1007/978-3-319-99701-8_2 . halshs-01953037

\section{HAL Id: halshs-01953037 \\ https://shs.hal.science/halshs-01953037}

Submitted on 12 Dec 2018

HAL is a multi-disciplinary open access archive for the deposit and dissemination of scientific research documents, whether they are published or not. The documents may come from teaching and research institutions in France or abroad, or from public or private research centers.
L'archive ouverte pluridisciplinaire HAL, est destinée au dépôt et à la diffusion de documents scientifiques de niveau recherche, publiés ou non, émanant des établissements d'enseignement et de recherche français ou étrangers, des laboratoires publics ou privés. 


\title{
Exercises in unstyling texts: Formalisation and visualisation of a narrative's [space, time, actors, motion] components
}

\author{
Jean-Yves Blaise, Iwona Dudek \\ UMR CNRS/MCC 3495 MAP, Marseille, France \\ \{jean-yves.blaise, iwona.dudek\}@map.cnrs.fr
}

\begin{abstract}
The research presented in this paper basis on the premise that segmenting textual content into successive situations according to four components - space, time, actors and motion - can help depicting a storyline in a way that facilitates comparative analyses across texts, and ultimately fostering knowledge discovery. The paper presents the original aim of the project and sums up the knowledge modelling choices made in order to formalise the segmentation procedure through which sequences of situations are extracted. We then present several proof of concept visualisations that facilitate visual reasoning on the structure, rhythm, patterns and variations of heterogeneous texts, and summarise how the space, time, actors and motion components are organised inside a given narrative. The approach was tested across various types of text, in three languages, and the paper details some of the potential benefits of the resulting visualisations on the specific case of R. Queneau's Exercises in style. The paper is concluded with a straight to the point analysis of the approach's actual weaknesses and limitations.
\end{abstract}

\section{Introduction}

A broad picture of the evolution of information sciences over the past decade shows that big data, meaning here big volumes of data, dynamically changing data, as well as high variety, highly heterogeneous data, has paved its way to the top of the research agenda. In parallel, availability of large collections of non-structured textual content, typically found in digital libraries, has fostered the emergence of research works clearly intermingling knowledge discovery issues with visualization issues.

A typical example of such ongoing approaches is the ReNom virtual library project [1]: “...the resource associates key texts by the two Renaissance authors [Rabelais and Ronsard] with a system of georeferencing and a series of fact sheets corresponding to the places and characters mentioned in the texts...”. In short, corpora of texts are explored in search of Named Entities (names of places, or of people) that can then be used as a means to navigate through the texts and to "... encourage a form of literary tourism at once fun and instructive...”.

This example does show that parameters space, time and actors can be a relevant entry point, a relevant filter, into text corpora. But the fact the approach bases on the extraction of Named Entities is a strong limitation: what happens when the space parameter cannot be associated with a given name, or is associated with a metaphoric 
name ("the silver ribbon in the distance" vs. "on the banks of River Loire")? What happens when an actor remains unnamed? With what geographical map can fictional places (e.g. the city of Barchester in A. Trollope "Barchester towers") be associated?

Furthermore such approaches are primarily designed as means to navigate inside text corpora, not across text corpora, and to localise elements in the flow of the text rather than to actually segment it. They provide no systematic, abstract overview of a text's structure that would enable comparative analyses - only the number of occurrences of a given Named Entity in a given text can be considered as a somewhat "generic" feature.

Finally, Named Entities are in the above example basically associated on one hand to positions in texts, and on the other hand to a "visual" component that can be either a map (localising a name of place) or an image (portrait of a person). The visualization components are designed as end-user services, displaying univariate data, and therefore do not play a significant role in terms of analytical tasks.

Our research is an attempt at going one step beyond in terms of genericity (encompassing texts that refer or not to Named Entities, introducing a segmentation method that covers various spatial scales, temporal markers or type of actors) and in terms of abstraction at the visualisation step (introducing a set of visual disposals allowing the analysis and cross-examination of multivariate data).

The title we have given to this contribution is a clear reference to R. Queneau's $E x$ ercises in style, one of the case studies that will be discussed. In that book the author demonstrates how for one same "story" (meaning one same succession of events, places, and people) there can be many ways to report how the story unfolds, there can be many ways to word out the story. But if that is so, couldn't there be a way to come back to a sort-of "root structure" of stories, enabling us to compare visually what they do have in common, and in what they differ? Wouldn't it be possible (and useful in terms of analytical tasks) to uncover and visualise that "root structure" - a storyline acting as the background of the story itself.

Our research can be seen as exploring one possible answer to these questions - a generic answer, applicable across various textual content, providing relatively satisfactory results, but definitely not the ultimate and unique answer.

It builds on the premise that a narrative can be segmented into successive or parallel situations differentiated from one another other basing on changes in time, space, actors, or motion. Each situation is therefore associated with four descriptors, and a change of value of one of these four descriptors is enough to trigger a new situation.

Such situations act as a semantic filter, helping to analyse and compare heterogeneous texts and collections of texts basing on common metrics. (Figure 1)

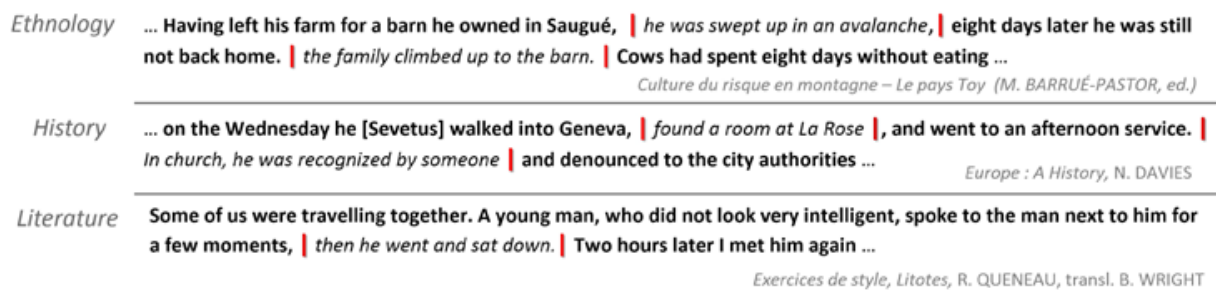

Fig. 1. Identification of situations in heterogeneous texts [2] 
Visualisations depicting sequences, rhythms, alternations of situations can then help experts and end users perform reasoning tasks on the narrative structure of texts, ranging from stylistic profiling (differences and similarities inside and across writing genres, or inside an author's works) to comparative analysis (different recounts of the same story for instance) (Figure 2).

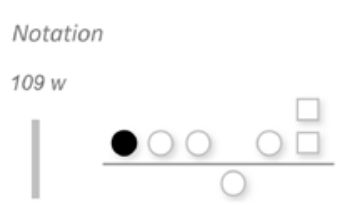

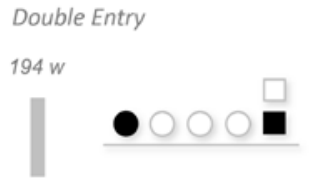

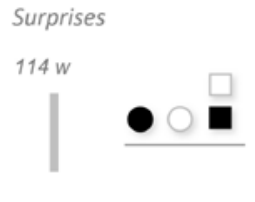

Fig. 2. A comparison of how situations unfold in time and space in three recounts of the same story (from R. Queneau's Exercises in Style). Note for instance that only situations (a, b) remain systematically the same across in these three versions.

The research unfolds in two sub-challenges a knowledge modelling challenge (How can we spot changes in space? What exactly makes a space to be differentiated form another - a name, a size? Who are actors - human beings only? ...) and a visualisation challenge (What visual solutions could help underlining expected or unexpected patterns inside or across texts?).

The paper is structured as follows: Section 2 introduces the reason to be of this research - it discusses the notion of situation that is at the heart of the approach. In section 3 we position our contribution with regards to existing approaches in the fields of visual analytics on one hand, and of text analysis on the other hand. Section 4 details our choices in terms of knowledge modelling, i.e. how the space/time/actors/motion components are used in the segmentation of textual content. Section 5 then presents a series of experimental visualisations corresponding to alternative combinations of variables and ultimately to alternative reasoning tasks. We then illustrate the potential benefits of these visualisations in terms of knowledge discovery for text analysis on one specific case: R. Queneau's Exercises in style. Section 6 describes the implementation and evaluation efforts carried out up to now. In section 7 we pinpoint strengths and weaknesses of the approach, and in particular challenges ahead if wanting to apply the approach on a large scale. Finally, a short conclusion section sums up what we think can be considered as fruitful feedbacks from this study.

The paper is an extension of a preliminary contribution entitled StorylineViz: A [Space, Time, Actors, Motion] - Segmentation Method for Visual Text Exploration [2].

\section{Research Issue}

\subsection{Origin of the research}

There is naturally a large range of features researchers may want to extract from text corpora, and analyse through visual means. Some are clearly structure-related, like in 
Marshman's [3] comparative analysis of lexical knowledge patterns. Others by contrast focus on spotting topics like Sabol's topical-temporal maps [4], a visual metaphor allowing an interactive analysis of how prominent topics in large collection of news releases change over time.

So why did we choose to focus on extracting the spatio-temporal content of textual data? The idea came as a natural continuation of years of research conducted on the architectural and urban heritage. Our usual concern, intersecting InfoVis (Information Visualisation) and Heritage sciences is analysing and visualizing architectural transformations, from the point of view of morphology (changes of shapes), from the point of view of chronology (duration, dating of changes), and from the point of view of events and people (correlation of data about changes) [5,6]. In short, we pull together a large amount of heterogeneous historical evidence, implement ad hoc information systems and ultimately provide researchers with means, inspired by the InfoVis legacy, to analyse this evidence visually.

The input we handle is therefore historical evidence: hints about space (e.g. an edifice, a garden, a marketplace), time (e.g. a period of construction), events (e.g. a war, a plague) and people (e.g. a ruler, a builder, an owner). At the root of our research processes there are physical (remains), visual (iconography) or written testimonies and pieces of data, that we consider from the point of view of what they tell us of a space, of a moment in time, of events and their impacts, of people that act in that space at that time.

A significant part of the historical evidence we use is extracted from texts, ranging from inventories to travel diaries and historical research, used for instance to "anchor" events and actors in time, space and context:

“... On Saturday, 12 August 1553, a fugitive from the holy inquisition rode into the village of Louyset..." (N. Davies, Europe:A History, Pimlico, London 1997, p. 493)

At the end of the day hints are recorded as corresponding to a given place, a given time, a given set of actors that altogether form a specific state.

Recounting and analysing an evolution can then be done by comparing successive "states", opening on a very general notion of 'path', understood as a series of situations leading from an initial state to a final state. This series is consistent or not in terms of spatial scale or quality of the information describing situations. It can be continuous or not (i.e. including or not temporal breaks).

\subsection{From reasoning on states to reasoning on situations}

The above notion of path can be used to interpret and structure (i.e. segment according to consistent division lines) a variety of heterogeneous historical evidence: travel diaries, witness reports, inventories, iconographic material, etc. But could it act as a potential semantic filter far beyond its initial field of concern - historical evidence?

When going through historical evidence quite often both place and time are likely to be partially, when not poorly, described - a document will for instance mention something occurring "on street $\boldsymbol{A}$ at the beginning of spring". Neither space, nor time are consistently defined inside sources, and across sources (varying precision, varying granularity). In that context a 'path' - understood as a series of potentially ill-defined 
situations - is obviously closer to the content of a narrative that to highly structured data sets handled in route calculations offered by GPS applications for instance. Hence the attempt we present in this paper to try and see to which extent such an approach to text segmentation could be fruitful, beyond its initial context of emergence.

\subsection{Segmenting narratives as series of situations}

StoryLineViz should be understood as a proof of concept study that aims at developing a generic approach to narrative analysis, supporting the identification and visualisation of significant patterns inside textual data, and ultimately knowledge discovery and sensemaking. Narratives as seen from that general point of view are strongly heterogeneous (from whole texts to just series of facts, from a book or collection to a few paragraphs). In addition, they can be contradictory or conflicting (different recounts of a series of events) or transformed (typically by translations). As of today they are often categorised (a play, a travel diary, an eye-witness report) and analysed from an expert's point of view (linguistics, literature, history, etc.) but hard to synthesize and to compare to one another.

In this contribution we propose an approach in which a narrative is segmented in a series of situations in ordinal time (i.e. only the order of appearance of situations is defined: situation $\boldsymbol{A}$ occurs before situation $\boldsymbol{B}$, but neither $\boldsymbol{A}$ nor $\boldsymbol{B}$ need to be actually dated). A situation is differentiated from another basing on the variation of one of the four following parameters: time, space, actors, and motion.

Our approach's core objective is to facilitate visual reasoning on the structure, rhythm, patterns and variations of texts in order to enable comparative analysis and to summarise in a clear-cut manner how the space/time/actors/motion components are organised inside and across narratives.

Quantitative and qualitative parameters can then be taken into account, allowing the association of causal or contextual indicators. The segmentation procedure is seen as a common ground between varieties of narratives. It aims at facilitating visual reasoning on the structure, rhythms, patterns and variations inside narratives or across collections of narratives. If proven workable the approach opens a number of application scenarios, among which:

- comparing oral or written recounts of the same series of events by different witnesses,

- comparing different recounts of the same itinerary (e.g. what differences can be spotted in the way pilgrims walk the Camino de Santiago today, and before, back to the initial Codex Calixtinus),

- $\quad$ supporting the identification of trends, patterns, evolution in writing genres in an edutainment-like approach (e.g. To which extent do classic Greek theatre plays and their 20th century reinterpretations overlap with the famous "unity of space, time and action” rule?),

- $\quad$ uncovering differences in the interpretation of texts by different readers.

At the end of the day, the approach can also be seen as an attempt to step out of discipline-specific frameworks so as to promote sort of "universal”, comparison-enhancing, metrics of narratives. However it should be said right away that this research 
makes no claim in the context of natural language processing or massive content analysis - what is presented in our contribution is basically an attempt to try and see if the specific segmentation bias we introduce could stimulate further research and lead to unthought-of observations on the structure of narratives.

\subsection{The concept of situation: legacy, and open challenge}

The idea that a narrative is (at least in part) composed of successive situations, corresponding to space/time/actors/motion components, is definitely not new.

J.R.R. Tolkien's The Hobbit starts as follows: “... In a hole in the ground there lived a hobbit." The narrative is triggered in time, indications about space (a hole), actors (a hobbit) and motion (no indication of movement in that sentence) are given.

The idea that a narrative can be presented, including visually, as a series of situations is also far from being new. Italo Calvino in his remarkable Collection of Sand comments on the "figurative narrativity" of Trajan's Column, along which Trajan's two wars in Dacia are recounted situation by situation through engravings. Situations follow one another (ordinal time) along a 200 meters long spiral, going from bottom to top. Each bas-relief corresponding to one situation contains indications about space and actors - Trajan before the imperial tent, a legionnaire digging a ditch, and so on.). In another essay Calvino comments on the development and use of ribbon maps over time a map including time and space, a map representing a path. A beautiful example of such maps is the mediaeval (12th century) ribbon map representing the "London To Jerusalem" itinerary by Matthew Paris, on which each stopover is represented by an outline of the city [7].

The idea to depict situations in a less figurative manner than in the above examples ins not new neither. For instance Historical centographs developed during the 19th century as a mnemonic system [8] introduce a level of abstraction, and an ambition for visual comparative analysis, that are much closer to our approach. Centographs represent time (ordered time model) though grids of squares: a 10x10 grid represents 100 years. Each square (representing one year) is then subdivided in a $3 \times 3$ grid, with each of the nine sub-squares representing a "variable" that can be chosen freely (lifetime of a person, event such as a war going on, etc.). Users of the system would fill in blank templates in order to underline co-occurrences in an attempts at facilitating memorisation tasks for students. This example does not precisely correspond to the notion of situation in narrative, but the visual solution can act as food for thinking: space/time/actors/motion are four "variables" that we observe in order to trigger a "situation change".

More recent, and situated in terms of graphic language somewhere in between the figurative nature of ribbon maps, and the strict abstract nature of centographs, is the Home to School diagram by A. Yabuuchi [9] where an itinerary is depicted as a series of situations each of which being associated with a set of "qualitative" variables (sound, type of landscape, type of vehicles, etc.). The resulting visualisation is a very elegant linear diagram looking more or less like a musical stave with each line bearing a variable, and the overall diagram clearly underlining consistent sequences in the overall itinerary, major trends, and exceptional situations. 
What can be said is that these examples do back up the idea that indeed a narrative can be segmented into a series of situations, reduced to sequences that somehow summarise the story, provide outlines, stop-points. But they give no hint at all on how to segment narratives, on which division lines should be used to decide whether or not a new situation has to be reported. Furthermore, they give no hint on how features corresponding to each situation (e.g. space, time, actors, and motion) can be compared to one another. For instance in the above example of Trajan's Column the initial situation, from the point of view of space is "the landscape of a fortified Roman Town", and the next situation "Roman soldiers crossing the Danube on a pontoon bridge". How do these "spaces" relate to one another? In section 4 we propose a strategy for associating each feature with a formal grid of descriptors designed as a mean to allow for crossexaminations : the "fortified Roman Town" spatial indication would be associated with scale 7 , and the "Roman soldiers crossing the Danube" with scale $\boldsymbol{8}$.

In other words our contribution is definitely not in pushing forward the concept of situation, but in the interplay between formal segmentation rules (enabling the identification of situations) and interactive visualisations (enabling a user-side analysis of situations and sequences of situations). Ultimately the challenge addressed here is to test a segmentation into situations that potentially says something about the structure of the narrative itself, or about the producer of the narrative.

\section{Scientific Context}

Open access to massive textual content, typically as found in digital libraries, has fostered the emergence of research works intermingling knowledge management, visualization, and language processing issues. In this contribution we focus on large nonstructured texts. Unlike when handling structured data sets, working on large texts, today often made available in large open access repositories such as Gallica, introduces specific challenges. Oelke [10] summarizes some of them: quantity (amount of words), polysemy (of words, references, literary imagery), flexibility (of rules in natural languages), interpretation (use of a predefined knowledge of the world by humans).

A typical example where space and actors are extracted from narrative texts is the CHAPLIN (CHAracters and PLaces Interaction Network) tool [11] - following a usermonitored extraction of terms graphs are produced that represent connections between places and people. Another significant example, this time focusing on temporal aspects, can be found in [12] - visual analyses of sentiments and character interaction in the flow of a fiction - an approach closer to what will be discussed in this paper but basing here again on the appearances and co-occurences of named characters in chapters.

Said briefly, there is a move towards bridging the gap between on one hand linguistics-based approaches - i.e. for instance spotting markers of cause-effect relations in text corpora, as in [13] - and on the other hand information visualisation approaches i.e. for instance tileBars for document visualisation [14], or basic wordclouds. Hence supporting text analysis through visual means has become a hot research topic in the field of visual analytics (VA), a field described in its early days by Thomas and Cook as "focusing on analytical reasoning facilitated by interactive visual interfaces" [15]. 
Our study proposes an approach that centres on semantic aspects, applicable across collections of texts. It builds on the idea that visualisation can help users explore, analyse and cross-examine textual documents. This idea is backed by research works covering a wide range of issues: VisRA tool [16] focuses on readability analysis, VarifocalReader [17] focuses on multi-layer visualisation/navigation and interactive annotation, POSvis [18] on relationships and co-occurences in the flow of a text, Wanner's approach [19] digs in the notion of opinion and sentiment in book rating.

Those examples share a common mantra: human analysis of textual content and sensemaking in large and/or complex textual data sets can be facilitated by adapted abstract visualisations. They also share a common statement: full automatic algorithms can hit their limit when facing complex texts.

Accordingly, our study does relate to the above research works in terms of scientific context, but it clearly leaves aside the NLP (Natural Language Processing) issues. We shall in this contribution focus on the knowledge modelling step on one hand, and on the visualisation step on the other hand. Mainstream research works at the intersection of VA and NLP have been investigating approaches that strongly rely on a line per line, word per word analysis of textual content: statistical approaches (e.g. occurrences of words, lengths, types of words), Named Entities Recognition (NER) related approaches (e.g. user selections of words, ontologies, opinion indicators), machine-learning approaches (e.g. extraction of significant linguistic patterns). In all these cases, language itself - i.e. the occurrences, positions, lengths, relations of words and sentences - is at the heart of a discipline specific analysis.

By contrast our approach builds on a segmentation bias that is:

- $\quad$ neutral - allowing for a discipline-independent cross-examination of texts,

- unrelated to text features such as lengths (a new situation can occur inside one sentence, or after three pages),

- focusing on supporting visual comparisons of rhythms and sequences, at userchosen aggregation levels.

As will be discussed in section 7, we do acknowledge that the language processing step remains at this stage of our research an unaddressed issue. The segmentation of texts used in the study has been done manually: it could be seen as a weakness in terms of significance and reusability of the approach.

We believe that before any attempt at "automatizing” language processing it is key to formalise a robust, insight-gaining, unambiguous segmentation protocol, and to evaluate in what the visualisations can be beneficial. Accordingly we consider that our study can contribute to pinpointing a new research path, at a time when the focus is often put on the processing of massive data sets.

\section{Method}

We introduce a text analysis method that builds on the identification of quadruplets of components: actors, space, time and motion. These components are used to segment a narrative and translate it into sequences of situations in ordinal time (only the order of events considered). (Figure 3) 


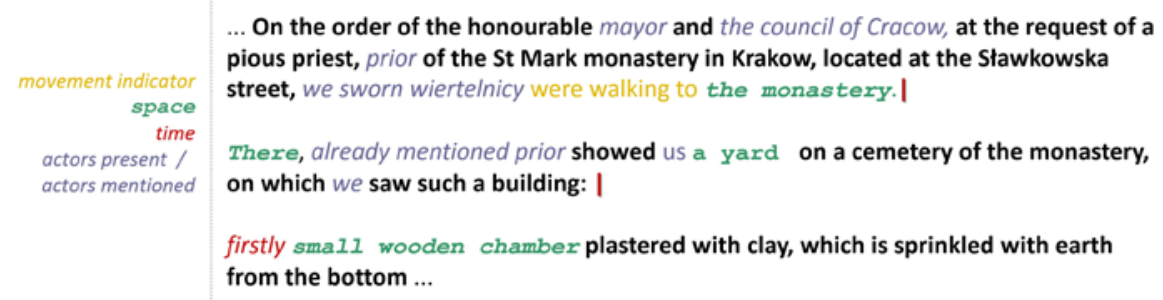

Fig. 3. Segmentation into situations - four indicators (Actum feria sexta ante Fabiani et Sebastiani [19I] anno Domini 1596). [2]

A situation is basically a sort-of token, resulting from the segmentation procedure. However we are here far from a segmentation at the word or sentence level: situations are determined by changes of values in a quadruplet of descriptors (space, time, actors, and motion). A change of one of the four descriptors introduces a new situation - (Figure 4). Situations occurring in the past of the story (e.g. reminiscences - narrating past experiences) are differentiated from those occurring in the course of the story.

- a change in space

e.g. Kate in a hospital room | She went out into the corridor.

- any break in the continuity of the story

e.g. She was unconscious as they carefully laid her back in her bed. I She woke a few hours later with a wintry sun seeping through the window.

- a change among actors (e.g. actors coming in or out)

e.g. Dirk went in.| Another policeman was standing in the hall and looked at Dirk blankly.

- a move from a static to a dynamic situation, when at least one of the actors is in motion

e.g. He stood there for a second or two longer| then he turned and stalked grimly back into the den of the beast.

Fig. 4. A segmentation procedure ending in the identification of independent situations basing on changes in space, time, actors or motion: example of application to D. Adams' The Long Dark Tea-Time of the Soul. [2]

Situations are identified at this stage through a manual annotation and segmentation process - a dozen of texts ranging from literature to ethnology have been tested, covering three languages. Each situation is associated manually with a value for each of the four descriptors, and with a short paraphrase summarising "what happens". The four values are translated into an alphanumeric code comprising indicators for each of four parameters and separators that allow for a processing of the information. (Figure 5)

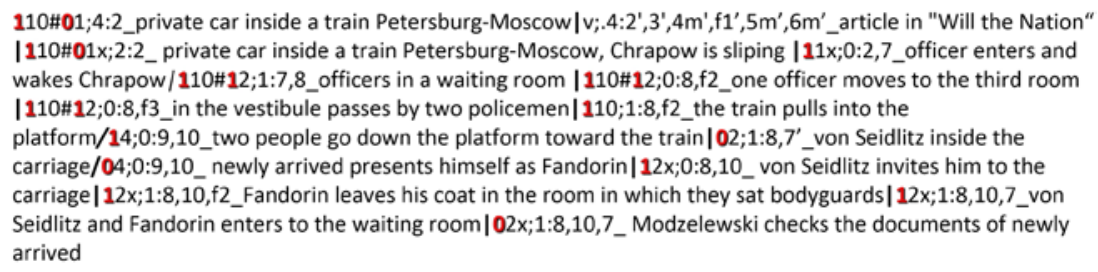

Fig. 5. Example of the alphanumeric code resulting from the annotation phase (in red, the code corresponding to the motion indicator - 0 static 1 dynamic - example from The Death of Achilles by B. Akunin). [2] 
Situations can also be grouped by predefined sequences such as chapters (or any other main division of a document). The way each component is defined and structured is detailed in the following sub-sections. At the end of the analysis phase the text under scrutiny is entirely transformed into sequences of situations as they occur in the narrative. Sequences are then translated into a visual language.

\subsection{Space Parameter}

The space parameter defines where the action takes place (i.e. Where does the action begin? Does it continue in the same location? Are the subsequently cited places well identified or in vaguely mentioned locations? Are their many quick changes of space? Are these changes related with a jump in time, a flashback for instance?). But 'space' as geographers, historians, architects, or ethnologists picture it is far from being one and only one notion. It can be described quantitatively (positions, size, exact morphology) or qualitatively (through linguistic indicators, or a relation to a Named Entity, for instance a toponymy like in [20]).

In the context of this study we need to spot in the flow of a narrative the moments when a change of space occurs, and therefore leads to a new situation (whether spaces are associated with a given named entity - e.g. Paris, or are present in the flow of partof-speech - e.g. in the second cellar). Detecting such changes implies defining unambiguous lines of division between spaces.

To do so, we reinterpret the concept of scale (in accordance with previous research on spatio-temporal information retrieval [5, 21-22]. What is meant by scale is not a map's numerical ratio, but the idea that spaces can be classified according to alternative spatial granularities.

Our model of space includes 16 indicators (3 non-spatial descriptors and 13 scale identifiers). The non-spatial descriptors concern the situations where space is not clearly assessed (metaphorical descriptions, undefined space, space is not present) -in other words non-spatial descriptors help dealing with incomplete, ill-defined, or simply missing spatial information.

The thirteen scale identifiers are organised into six groups (e.g. in and around a building, public spaces, open land). An additional parameter is taken into consideration: primary vs. nested spaces. Primary spaces correspond to 'simple' situations (e.g. Jane is in her room, Jane is walking in the garden). Nested situations appear when actors are inside vehicles or objects that can move or be moved inside a primary space (e.g. Jane is travelling by train.).

\subsection{Time Parameter}

The time parameter corresponds to the when question: it explains the story's development over time (e.g. continuous progression from present to future, regressive presentto-past development, multiple changes of time, etc.).

The time model builds on the notion of ordinal time [23]: situations are analysed from the point of view of an order of appearance (before/after) in the flow of the narration, but neither quantified nor anchored. (Figure 6) 
initial situation >

new situation, short lapse of time >

new situation, longer lapse of time >
Having left his farm for a barn he owned in Saugué [...]

He was swept up in an avalanche [...]

Eight days later he was still not back home [...]

Cultures du risque en montagne M. Parrué-Pastor (dir.) L'Harmattan

Fig. 6. Change of situations - temporal disruptions

A qualitative assessment of time continuity is associated to each situation change (lapse of time separating a situation from the next one).

Successive situations are identified in the order of the narration (as the story unfolds) as belonging to the present of the story or its past (things having occurred "before the present of the story"). Situations can also be tagged as being parallel (occurring at the 'same' time). (Figure 7)

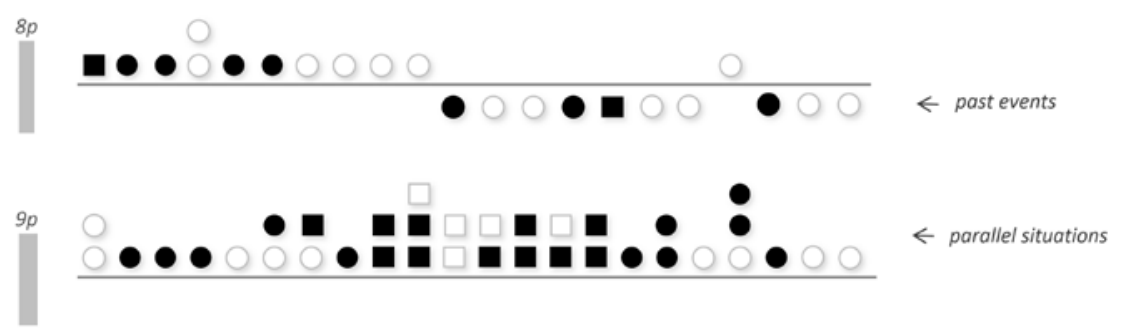

Fig. 7. Top, past situations are represented below the horizontal line. Bottom, parallel situations are represented by graphic elements “piled” one over the other above the horizontal line. [2]

Additional indicators are used to further describe parallel situations (actors mutually aware of one another or not, typically), or to identify customary behaviours (occurring repeatedly).

In the context of this study we need to spot in the flow of a narrative the moments when a change of space occurs, and therefore leads to a new situation (whether spaces are associated with a given named entity - e.g. Paris, or are present in the flow of partof-speech - e.g. in the second cellar). Detecting such changes implies defining unambiguous lines of division between spaces.

\subsection{Actors}

Actors are yet another trigger of situation change. They may be individuals, well defined groups of people, but can they also be indistinctly specified groups (e.g. a crowd), things (e.g. thinking machines), or animals? We here need to disambiguate the very concept of actor: are ants mentioned in B. Werber's Empire of the ants actors?

Our strategy is to consider actors as a being or a consistent group of beings, real or imaginary creatures or entities, fitted with the ability to make choices and to act. Actors may be human beings, but also gods (e.g. Zeus, Dionysus), thinking machines, androids, animals (e.g. the wolf in Little Red Riding Hood), and so on. The description of 
actors is then fine-tuned. Actors physically engaged in a situation (i.e. present) are distinguished from actors that are only mentioned (e.g. in a conversation, or in thoughts), individual actors are distinguished from consistent groups either identified (e.g. the Celts) or not (e.g. a crowd). (Figure 8)

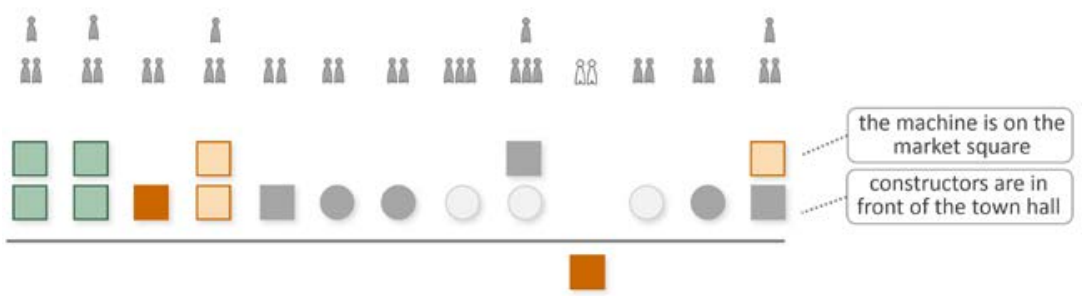

Fig. 8. Actors appearing in each situation of S. Lem's Trurl's Machine. Situations are read from left to right. Here Trurl and Klapaucius, the two engineers (bottom part of the lines showing actors as silhouettes) are being chased by Trurl's machine gone mad (top part of these lines, one silhouette alone). A reference to past events is made (orange square below the horizontal grey line), and that past situation concerns two actors not present but mentioned (white silhouettes).

Finally, major events concerning actors can also open up on a situation change - a severe injury, or a death of an actor needs to be reported.

\subsection{Motion}

Finally, motion is also a key element in the definition of a situation (only the motion of actors is considered). Motion is important to state since it helps unveiling spatial and temporal continuities or discontinuities in the narrative. An intensive use of motion indicators in a text may characterise writing genres (e.g. logbooks), may underline recurrent stylistic elements (e.g. a speed chase with the police), stylistic characteristics of an author, differentiate acts inside one play, help understanding changes in space, and so on. (Figure 9)

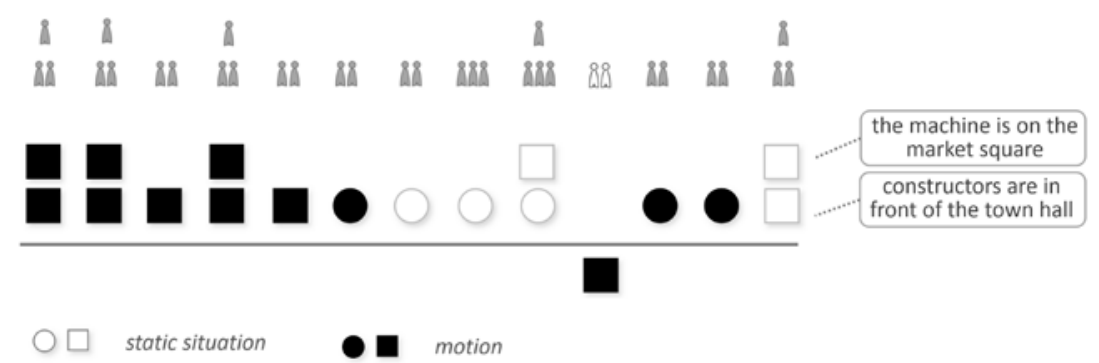

Fig. 9. A partial view of motion analysis visualisation corresponding to S. Lem's Trurl's Machine. Light grey elements indicate static situations (e.g. the engineers discuss with the mayor of a town in which they sought refuge).

Naturally we need to be clear on what we mean by motion. The strategy is to focus on movements that introduce a change of location but not necessarily a change of space 
(e.g. someone is walking down a street). From the point of view of this criterion situations may then be classified as static or dynamic. A dynamic situation implies the motion of at least one of the actors, motion understood as moving in space (e.g. walking, marching, strolling, running, driving a car...).

\section{Visual Solutions}

Our approach bases on the idea that interactive visual interfaces can help various target users perform reasoning tasks in application fields ranging from expert analysis to education or cultural mediation. Accordingly visualisation is a key component of the study, both in the understanding of a given narrative's "spatio-temporal profile" and in fostering comparisons inside collections of texts. What is meant here by the term visualisation is made clear by V. Sabol [24]: (a) graphical representation of data, information and knowledge visualisation, (b) using the human visual system, supported by computer graphics, to analyse and interpret large amounts of data, (c) visual representation to aid cognition.

Depending on the parameters a user may choose to privilege (i.e. space, time, actors or motion), different visualisations are proposed. We detail them in the following subsections. All of these visualisations share some common design principles and a graphic language that we try to adapt to a human's visual apparatus limited number of preattentive features [24]:

- Situations are represented one by one and aligned as they occur in the original text (left to right, or top-down),

- Each situation is represented by an interactive symbol (a multidimensional icon). Shape, colour and position are used to transfer visually the information on each situation,

- A rephrasing of the actual text corresponding to each situation is available on user demand,

- Parallel situations, i.e. situations co-occurring in time, are grouped and represented together,

- Actors are visualised on user demand, with colours differentiating the nature or type of actors (actors present, mentioned, injured, or groups of actors).

Situations are grouped by sequences (chapters or other grouping mechanism adequate for a particular writing genre) in order to grab more easily an understanding of the text's structural features.

\subsection{Spatial Sequences Visualisation}

In the spatial sequences visualisation situations are represented in ordinal time from left to right along horizontal bars. Each horizontal bar corresponds to a sequence of situations. All reminiscences are situated below horizontal bars (Fig. $10 \boldsymbol{b}_{1}, \boldsymbol{b}_{2}, \boldsymbol{b}_{3}$ ). Colour and shapes are used to differentiate the occurrences of various spatial scales. 


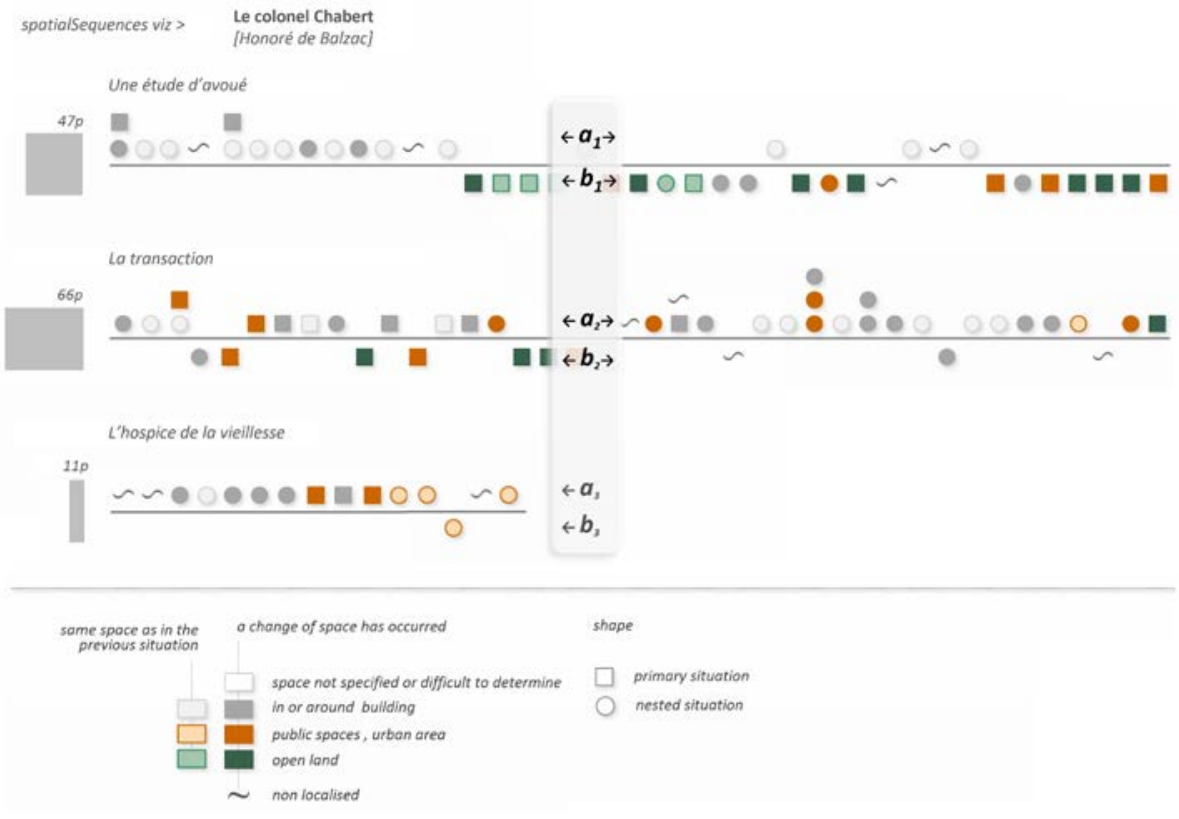

Fig. 10. Organisation and legend of the spatial sequences visualisation. Bottom, legends of the visualisation - colours correspond to ranges of scales. Squares and circles differentiate nested spatial configurations (e.g. driving a car in a city) from primary configurations (e.g. walking in a city). Top, a partial view of the spatial sequences visualisation corresponding to Balzac's Colonel Chabert. Note for instance the contrast between spatial location of present (a1) and past (b1) of the story in chapter one (colours), or the quasi-absence of past events in chapter 3 (b3). [2]

\subsection{Motion Analysis Visualisation}

The motion analysis visualisation uses the same general organisation as the previous: situations are represented in ordinal time from left to right along a horizontal bar. But here the focus is put on the motion component of the model: colours and transparency representing different types of space are replaced by black-and-white motion indicators.

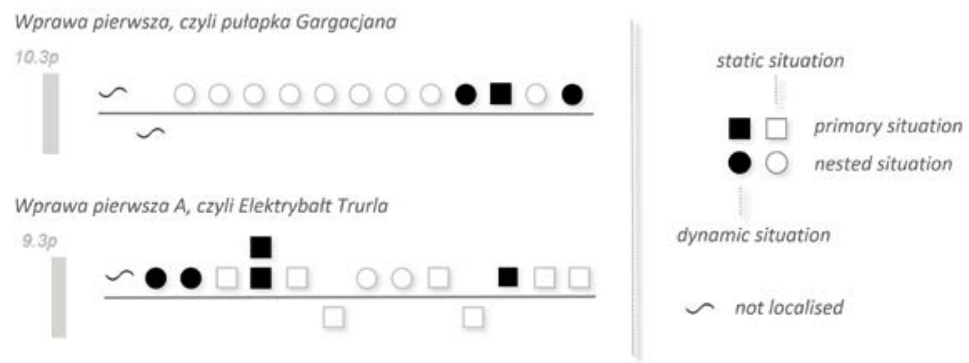

Fig. 11. Organisation and legend of the motion analysis visualisation. Left, a partial view of the visualisation corresponding to S. Lem's Cyberiada. Note for instance the long sequence of static, nested situations in chapter 1 . Right, legend of the visualisation. [2] 
This visualisation is used to differentiate static and dynamic situations, thereby better underlining in particular rhythms inside a text. (Figures 7,9,11,14)

\subsection{Temporal Continuity}

The temporal continuity visualisation focuses on assessing visually to which extent the story unfolds without interruption in time. (Figure 12)

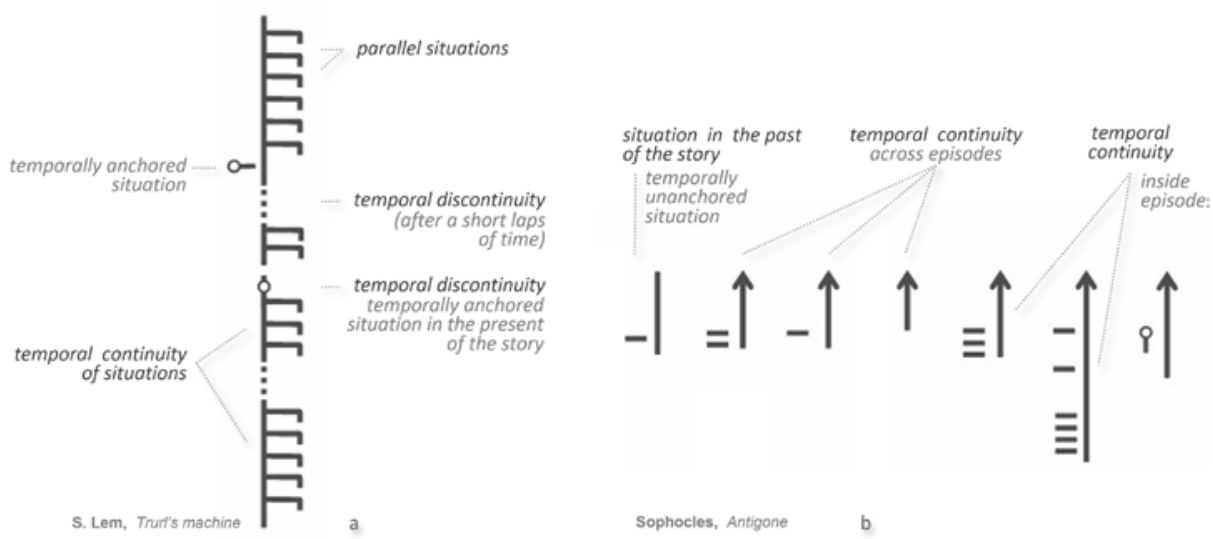

Fig. 12. The temporal continuity visualisation: (a) applied to S. Lem's Trurl's Machine. The visualisation shows an intensive use of parallel situations, and spots three lapses of time disrupting the tem-poral continuity, (b) applied to Sophocles' Antigone, the visualisation illustrates the unity of time pattern - a typical example of classical unity of time rule for drama.

Each sequence (i.e. chapter, episode, etc.) is here represented as a vertical line. A line topped with an arrow shows a temporal continuity with a previous situation. Small horizontal lines distributed on the left side of the vertical line correspond to situations occurring in the past of the story. Parallel situations are identified by symbols positioned on the right side of the vertical line. The vertical line is disrupted by various symbols in cases of temporal discontinuity (different symbols are used to represent short lapses of time, jumps in time, temporally unanchored events, etc.).

\subsection{Spatio-Temporal Continuity}

The spatio-temporal continuity visualisation builds on the same design as the previous, but adds symbols representing the space parameter. Whereas in the spatial sequences visualisation (section 5.1) we only deliver an indication about the group of scales corresponding to a situation, we here allow for a visual coding of each of the thirteen individual scales. Fine-grain differences can be made for instance to differentiate a situation occurring inside a building from a situation occurring in a building's courtyard, or in a flat forming part of the building. (Figure 13) 


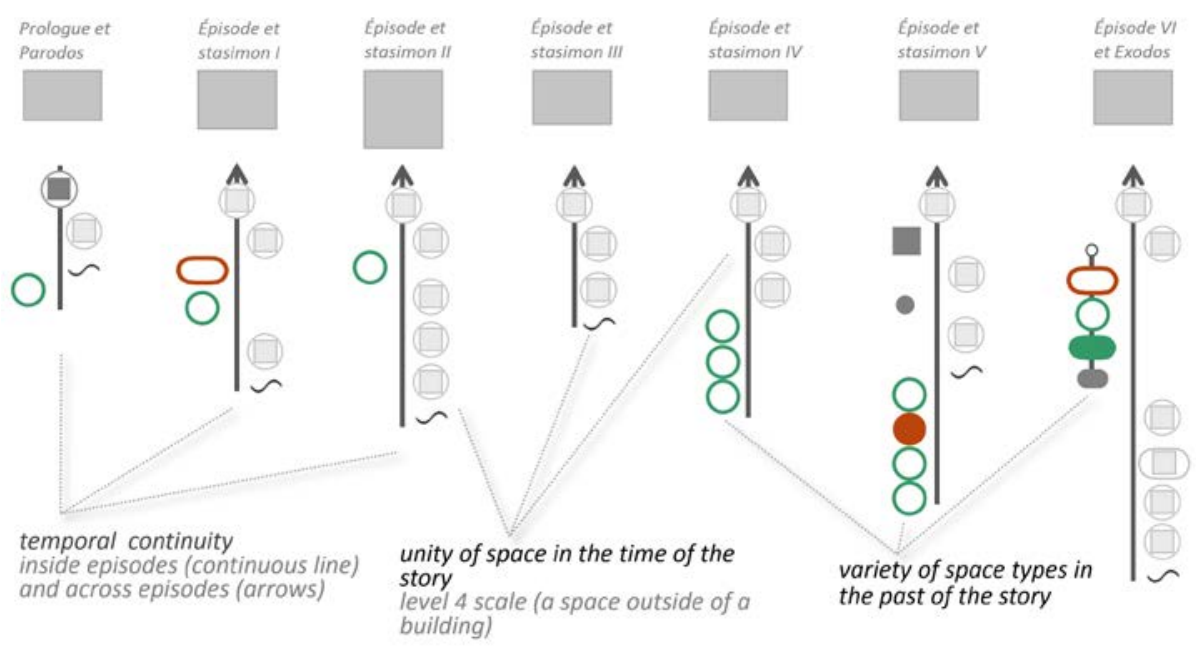

Fig. 13. Spatio-temporal continuity visualisation corresponding to Antigone of Sophocles. Note contrast in terms of space between the past of the story (symbols on the left of the vertical lines) and the present of the story (symbols situated on the vertical time-line and on the right of it). Note also that in the present of the story space remains unchanged (in front of the palace).

\subsection{Illustrating the Approach on a Case Study: Exercises in Style}

Raymond Queneau's Exercises in Style is a 1947 book in which the author retells the same story 99 times, each time in a different "style". The word "style" should however here be interpreted as something of an over-simplification when reading the exact titles given to the versions: "metaphorically", "precision", "logical analysis", "philosophic", "official letter", etc. What Queneau does really is demonstrating there is a distance between the events reported and the wording. Briefly said the story is this of someone who gets on a bus, witnesses an altercation between a long-necked man and another passenger, and then notices this same long-necked man two hours later close to a railway station and getting advice on adding a button to his overcoat. Because it clearly questions the distance between facts and reports of facts, between what makes "style" a layer "above" the meaning of what is said, Queneau's work was obviously a promising test bench for our approach.

And indeed, as is shown in the examples proposed below, the series of events reported in each of the 99 versions is not exactly the same : the storyline remains, but there are here and there situations that are added, withdrawn, or modified in terms of what is known of each situation. Because the method we explore involves exclusively relations between time, space, actors and movement, we naturally make no claim that the segmentation procedure we propose, and the visualisations we have tested, do cover the entire subject matter of Queneau's illustration of the distance between a storyline and its wording.

But the following examples show our approach does help pointing out significant resemblances and contrasts between versions, trends and exceptions in the whole collection of 99 versions - i.e. encourages analytical reasoning on textual content. 
Highlighting differences. This first series of visualisations show the approach does help highlighting differences between the various versions of the story. Some sharp contrasts can be noticed in terms of quantities (number of situations, number of situations that include motion, etc.) but also in terms of "stylistic figures" (use of recollections, parallel situations, metaphors, etc.).
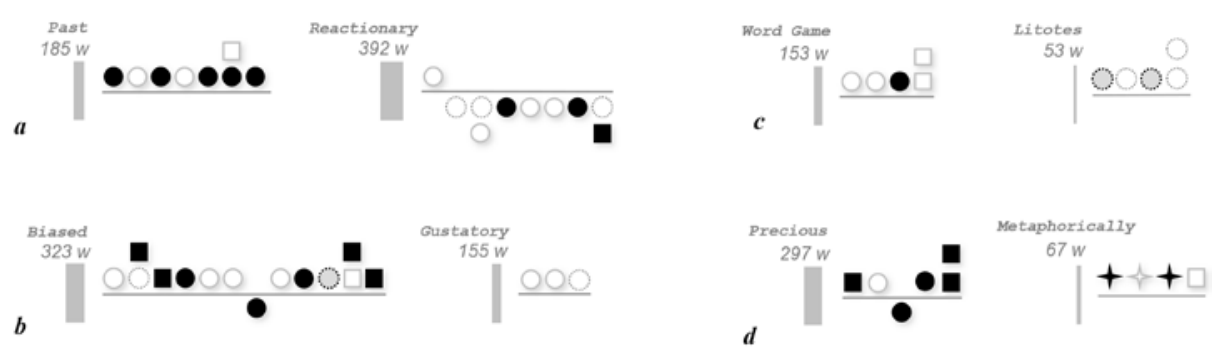

Fig. 14. Motion analysis. Two by two comparisons underlining differences between versions: (a) story told in the present vs. as a recollection; (b) high contrast in number of situations; (c) spaces named $v s$. unspecified; factual $v$ s. metaphorical recount

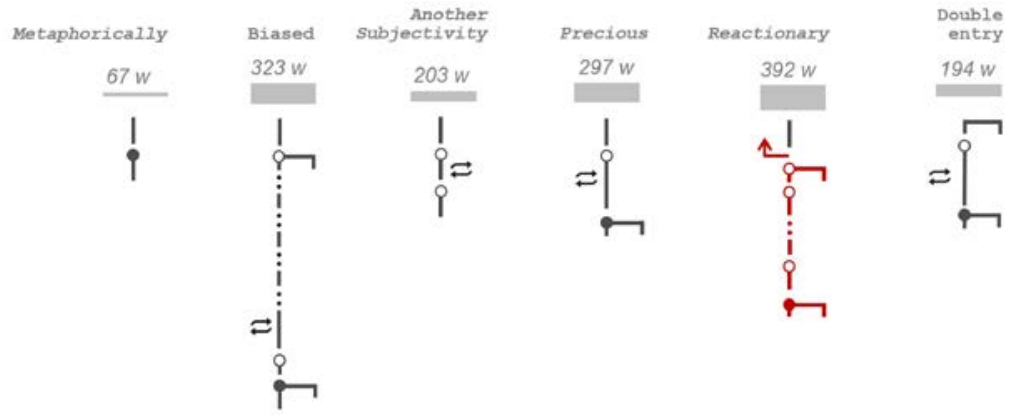

Fig. 15. A comparison of temporal patterns across 6 versions showing a strong variety.

Spotting groups and patterns within groups. The fact that stories differ from one another is not surprising - the visualisations in that case basically back up something that is rather intuitive for readers, and give some "factual" basis to that intuition. But the fact that within the collection there are consistent groups of stories (in terms of space/time/actors/motion components) and that within these groups there are some specific patterns just cannot be noticed naturally, intuitively. Yet such groups, and patterns, are clearly spotted in the examples below.

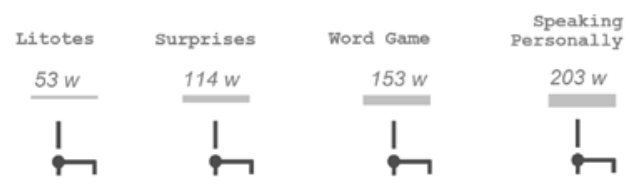

Fig. 16. Four temporal continuity visualisations corresponding to four versions that are strictly identical from the point of view of time, thereby forming a consistent group independently of the lengths of the stories (counted in words, represented by the greyish rectangle above the glyphs). 


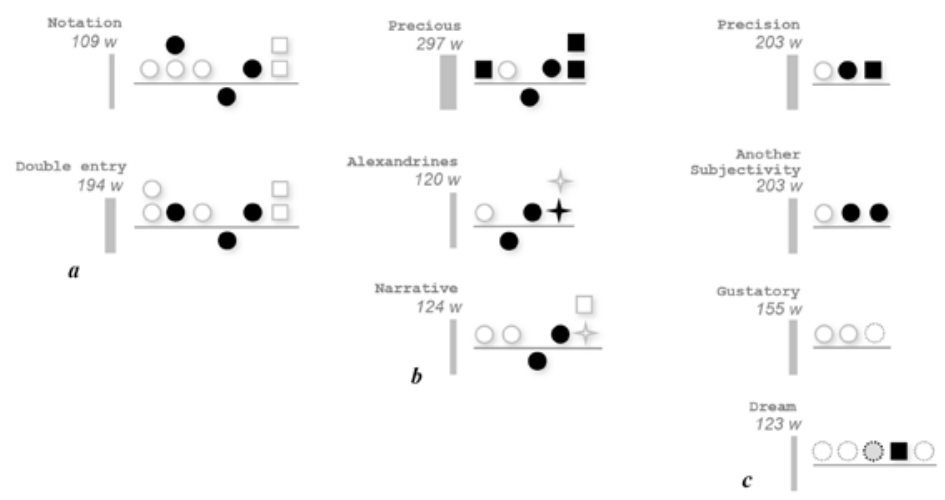

Fig. 17. Motion analysis visualisations corresponding to three groups: (a) two versions with almost the same number and type of situations; (b) different types of situations but a final situation always parallel, and a situation in the past always with motion; (c) a group of version with a rather limited number of situations, all in the present of the story, and no parallel situations.

Different stories, but invariant features. The visualisations also help spotting across the set of stories what can be called invariant features - typically the initial and final situations emerge as such.
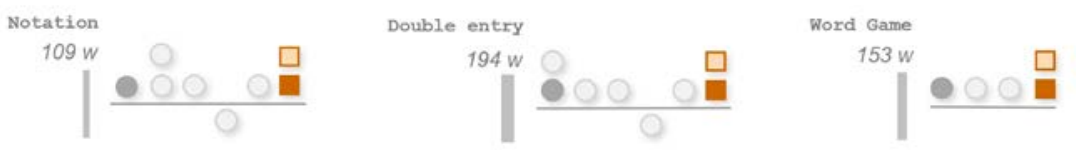

Fig. 18. These spatial sequences visualisations show a clear invariant feature in three versions: final parallel situations with the same indicators of space (both situations in cour de Rome).

Number of words, number of situations. One could probably think that the more words in the story, the more chances to meet new situations. Or one could think these quantities are unrelated. The visualisations show some interesting resemblances between versions in terms of number of situations and number of words. But they also clearly show that the number of situations is not correlated to the number of words, rather to stylistic choices (presence of verbose descriptions of places or people for instance, or of feelings and thoughts).

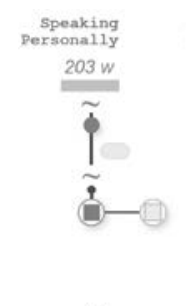

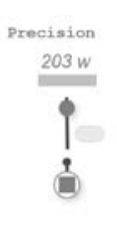

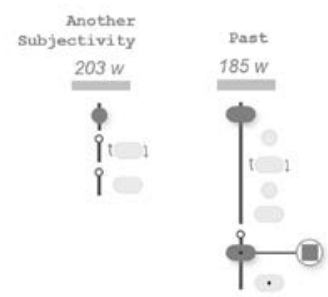

$b$
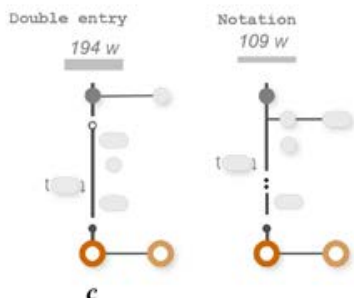

Fig. 19. Six spatio-temporal continuity visualisations: (a) same length, and almost same number of situations; (b) longer text on the left, higher number of situations on the right; (c) almost similar diagrams but contrasting lengths. 
Fine-grain comparative analyses. A number of fine-grain one to one comparisons and analyses can be carried out on versions of the story by observing for instance the spatial granularity used to tell the story, the temporal disruptions introduced, the exact moment of appearance of the same event in the various versions, etc. (Figure 20).
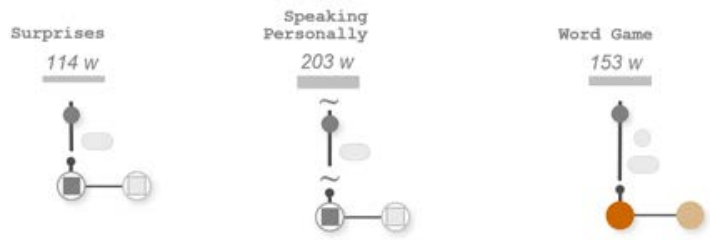

Fig. 20. Spatio-temporal continuity visualisations. The number and type of situations in version Surprises and Speaking Personally is almost the same, but the number of words differs significantly, due to the two temporal disruptions marked by a tilde (personal remarks and opinions by the narrator on general topics). Speaking Personally and Word Game stories are quite close but the former positions the last situations (parallel situations) in front of a building (the railway station) whereas the latter positions them somewhere on an urban square (cour de Rome).

\section{Implementation and Evaluation}

The approach has been tested on different types of text: a play (Sophocles), crime stories (A. Christie, B. Akunin), science fiction and fantasy (S. Lem, T. Pratchett, D. Adams), French literature (H. Balzac, R. Queneau), reports of interviews (e.g. ethnological research) or historical texts (e.g. 16th century textual building inventories).

The corpus includes textual content written in English, French and Polish. One of the reasons of this choice was to check that the approach is workable in different languages. Another reason was to test the impact of a given natural language - i.e. test if the segmentation of a given textual content, once translated into another language, remains fully consistent with the original.

As mentioned in section 4 the annotation step results in alphanumeric codes associated to each situation. These codes, along with bibliographic data and other general information concerning the texts, are stored in an RDBMS structure. They are interpreted on the fly (Perl scripts) to produce SVG (Scalable Vector Graphic) interactive visualisations available inside standard web browsers.

\subsection{Evaluation}

An early "feasibility" evaluation was carried out with a group of non-experts (twelve students in mechanical engineering) in order to get a first feedback on the knowledge modelling bias (segmentation into sequences of situations). We asked testers to depict an everyday series of actions, such as their home to work routine, using the graphical codes. We then asked then to complement the description of each individual situation with one or several qualitative parameters of their choice. Some recurrent parameters emerged, such as sound, amount of light, mood, etc. What this evaluation procedure 
did usefully underline is that the logic behind the segmentation protocol is easily understood, and somewhat intuitive.

Yet there is a clear difference between asking testers to analyse one of their own everyday routine in terms of series of situations and having them uncover these situations from a textual content using predefined segmentation rules. In a second round we therefore implemented a more demanding evaluation setup, with this time eight testers from different countries (Marie Curie fellows focusing on reality-based 3D modelling - no native speakers of English) working on two extracts from novels written in English. It has to be said right away that the fact that testers were not native English speakers and the fact that the whole evaluation process (including the introduction to the approach, the reading of texts, the segmentation effort itself) lasted two hours and a half only undoubtedly minor the scope and significance of the evaluation and relativize the conclusions that can be drawn. Yet as will be discussed below the experience has impacted the way we can foresee future developments of the approach. It in particular helped us pinpoint a noticeable difference between perceptions of spatial disruptions (relatively consistent among readers) and temporal disruptions (very erratic). It also helped us understand that beyond what we considered at the beginning of this research as a key potential benefit of the approach (a systematic, discipline-independent segmentation and visualisation procedure) there is another, maybe even more promising benefit: helping different readers to formalise their own understanding of a narrative and thereby facilitating and structuring workgroup discussions.

The testers were first introduced to the approach, and shown the whole set of segmentation rules. Following, they were asked to work on a first text that they had to segment under supervision. This step was needed to make sure that the protocol was clear enough for them. These two phases lasted for an hour and a half. Testers were then left for one hour with a 1000 words text that they had to segment on their own, i.e. on one hand they had to spot situation changes and on the other hand they had to qualify each situation with regards to space (what scale?) to time (any disruption?) actors (who is concerned?) and motion (do actors move?).

A central issue we wanted to raise was whether or not situations, can easily and unambiguously be differentiated from one another. We analysed both the raw, quantitative results (number of situations spotted, types of scales identified, quantity of switches between static and dynamic situations, etc.) and the oral remarks made by the testers at the end of the evaluation.

Results show that generally speaking the concept of situation is quite easy to use testers had no particular difficulty in spotting different situations and tagging them with values for the four parameters. But if the mechanism was found clear, we spotted a number of ambiguities deriving from two different issues: comprehension and individual interpretation of the segmentation rules on one hand, and inherent "fuzziness" of texts on the other hand. The open discussion that followed the gathering of the oral remarks was a particularly stimulating moment. Testers started comparing their own understandings of the text and explicit their interpretations -for example, how they understood spatial indications given in the text about a situation at a railway station and why they selected this or that scale from our theoretical model. In that example it turned out that testers used recollections, images of one specific railway station and based their choice on this specific example. 


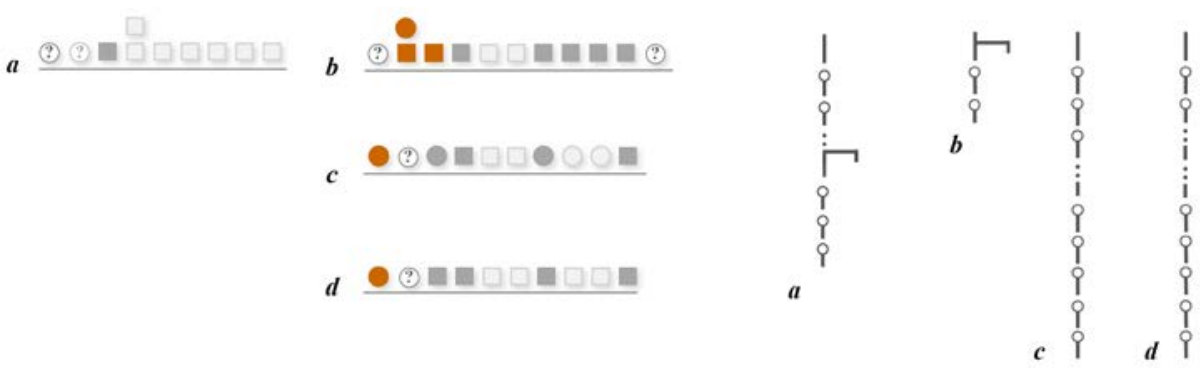

Fig. 21. A comparison between (a) results of the segmentation as we performed it and (b,c,d) results achieved by some testers, illustrated on the spatial sequences visualisation (left) and the temporal continuity visualisation (right). Differences in the spatial sequences visualisation are relatively limited in terms of number of situations spotted (9 vs. 10 vs. 11), but a little more significant in terms of scale identification: testers systematically tag one situation at least as corresponding to the urban scale, and several situations are tagged as "in a new space" (dark grey) when we considered space as unchanging in the text. Differences in the temporal continuity visualisation are by contrast quite sharp: testers understood, interpreted, temporal discontinuities in the flow of the narration in very different ways.

Comprehension Issues. The evaluation showed us that the testers had some difficulties with time discretisation and scale identification. Although testers globally understood the rules, they did not have enough time to get familiar with them before the test - they somehow discovered them as they progressed in the segmentation of the text.

We also noticed different individual interpretations of the segmentation rules: e.g. what does 'after an instant' really mean? Testers disagreed on this very notion. What kind of space is 'a railway station'? A building, a building and its surroundings, an inside, an outside? Here again each tester pictured what 'a railway station' is his own way.

A certain number of segmentation rules as we had verbalised them turned out to be either too loosely defined, or too interpretative - typically the notion of parallel situations that encompasses someone spying on others from behind a window to a phone call connecting two people located in different parts of the world. One type of parallel situations appeared as particularly confusing, when several groups of people are in the same space but act independently of one another - in this case the rule itself needs rethinking.

Finally, some testers questioned the segmentation rules themselves when the rules, according to them, did not let them stick close enough to the text. In the text proposed Kate is driven to the airport in a taxi - but no mention is made of the taxi driver in the initial situation. Those testers considered that putative actors - here the taxi driver should be mentioned, although according the segmentation rules they were given only actors mentioned in the text should be specified.

Briefly speaking, the evaluation showed that segmentation rules and definitions of scales, temporal disruptions, motion and actors need to be further clarified and illustrated by examples in order to pin down the concepts and reduce existing ambiguities. More generally the above comprehension issues clearly underline the fact that more time should be spent on explaining the segmentation rules prior to the evaluation itself. 
Moreover applying correctly the segmentation rules requires a thorough understanding of how space and time are discretised - which implies a steep learning curve.

At this stage the approach requires from readers and annotators a good understanding of the segmentation rules, but also keeping a certain "distance" with the text in order to avoid confusing what is really written, with what one may deduce, understand or imagine. What we asked the testers to do - segmenting of a text into an alphanumeric code using a set of segmentation rules and of discrete values - requires from annotators skills and capabilities. It definitely is a demanding task that limits the circle of people who can be expected to carry out the annotation step.

Inherent Fuzziness of Texts. There are a number of factors that impact the way space, time, actors and motion are verbalised by authors. Texts are written with a significant amount of unsaid, or half-said elements - voluntary omission of details, figures of speech, etc.

Consider this yet straightforward example: "She set off in search of first a newspaper and then some coffee. She was then unable to find a working phone". Should the reader here consider time as continuous, or as interrupted for a short while, for a long while? The author does not say openly whether there is a time disruption or not. The same can happen when mentioning spaces, actors, or even motion. Texts are the way they are, and readers will anyway interpret and understand them differently, whatever semantic-based segmentation rules one may write - a feature of what A. Korzybski named verbal levels [25].

The evaluation showed the inherent fuzziness of texts can be seen as an obstacle, but also as a potential object of study, an opportunity for instance to use the segmentation rules in order to localise areas where readers interpret a text differently.

Interpreting the evaluation's results should however be done with caution. The segmentation's learning curve is definitely steep: further evaluation efforts are therefore clearly needed (for example finding a match between a text and a visualisation in a setup where several possibilities are shown). It also underlined unexpected potential benefits of the approach, in its current state of development:

- It helps comparing how different people understand and interpret the spatio-temporal content of a text.

- It enhances debate, and helps uncovering precisely (in the flow of the text) where alternative interpretations occur, and why.

- It facilitates the communication by one individual of his own understanding of a text by supporting (through visual means) his discourse on rhythms of a narrative in a context + focus manner;

- It could be used to weigh and compare the level of interpretation required from readers depending on the text or author.

\section{Limitations and Perspectives}

StorylineViz should be understood as a proof-of-concept study, aimed at developing a generic approach to narrative analysis, but with at start no other ambition than investigating to which extent coupling a specific segmentation bias (space/time/actors/motion) 
with visualisation solutions could renew the way we can understand, compare, debate textual content. Hence we do acknowledge this research's actual impact needs to be weighed with regards to a number of significant limitations, and notably the following:

- We consider that the corpus of texts used as test cases is representative in terms of variety, heterogeneity, but it definitely is a partial corpus.

- The evaluation phase should clearly be deepened - notably with regards to finetuned usage scenarios.

- The comprehensibility of the segmentation rules for a wider public should be better assessed, as well as the learning curve.

- The implementation is a robust one, but it certainly could be rethought or improved.

In addition to these general remarks, and anticipating potential perspectives of the research, two major challenges ahead need to be pinpointed : working on the end-user service (visualisations), working on the upstream process (segmentation and annotation of the texts).

\subsection{Visual Reasoning: Still a Challenge}

Our approach bases on the idea that the combination of a non-standard segmentation procedure with appropriate visualisations can offer users new opportunities to perform reasoning tasks, and uncover pieces of knowledge inside textual content. But such a statement can only be corroborated (or invalidated) if the experimental setup proposed to testers is fully satisfactory. The visualisations we ended on do show the idea is worth exploring, but the implementation is at this stage not fully satisfactory. For instance support for a visual cross-examination of texts "within the eyespan" [26] needs to be improved. Accordingly we consider that our study needs to be extended and deepened in order to state without doubt that the approach is indeed, generic, workable across various types of texts, and fruitful in terms of knowledge discovery.

\subsection{The Impact of Manual Annotation}

Even more significant in terms of limitation of the research's potential impact is the fact that the annotation process - i.e. the segmentation of texts - is to this day a manual process. This clearly undermines perspectives of application of such an approach on a large scale. But on the other hand it also opens a clear perspective (and challenge) for this research. The approach hits the limits of existing NLP based methods. Hence rounds of discussion we are at this stage having with VA and NLP partners to try and investigate how the approach could be developed on a large scale. Even if a fully automated annotation process would turn out to be out of reach, working on semi-automatic procedures in the context of the emergent crowdsourcing paradigm would clearly open tangible large-scale application perspectives. Furthermore, human annotation is by itself a meaningful activity, opening perspectives in terms of communication and comparative analysis of text interpretation. Both going towards more automation in the segmentation process, and sticking to a human process, can therefore be considered as lines of development of the approach. 


\section{Conclusion}

In this contribution we present a research that aims at investigating how performing analyses of narratives (in a broad sense, encompassing texts that range from ethnological records to fictional stories) can be renewed by introducing an unusual segmentation bias and a series of ad hoc visualisations conveying insights on how the space, time, actors and motion components of the text interact in successive situations as the narrative unfolds. The main claim behind the approach is that extracting the spatio-temporal content of a narrative and visualising it as a series of situations can help spotting and exploring significant patterns, trends, exceptions across various types of texts.

In short, the main benefit expected from applying the approach can be summed up in two words: knowledge discovery. The corpus on which the approach has been tested remains partial, but the experimentation does show the approach is workable across various types of texts. Illustrated in this contribution on the specific case of R. Queneau's Exercises in style, it proved useful in uncovering unexpected and noteworthy patterns inside the 99 versions of the same series of events the book is made of.

The evaluation carried out paved the way towards usage scenarios that would focus more on assessing differences between reading and comprehension experiences than on the "automatization" of the segmentation process.

The visualisations produced until now show an interesting interpretative potential. They could be used for example to support teaching and learning activities, helping learners to quickly get a hold on patterns, trends, exceptions, and to carry out comparative analyses across texts (for instance using the approach in order to support pupils with learning disabilities such as dyslexia). We consider that, at this stage, the approach has proven workable, but will need further improvement loops (more case studies, more rounds of evaluation) before becoming fully operable.

\section{References}

1. ReNom: Navigating the Works of Rabelais and Ronsard in Search of People and Places. http://renom.univ-tours.fr/en/project visited 01-03-2017

2. Dudek, I., Blaise, J.Y.: StorylineViz: A [Space, Time, Actors, Motion] - Segmentation Method for Visual Text Exploration. In: Fred A, Aveiro D, Dietz J, Filipe J, Bernardino J, Liu K (eds) Proceedings of the 8th International Joint Conference on Knowledge Discovery, Knowledge Engineering and Knowledge Management, pp. 21-32, Volume 1: KDIR, SCITEPRESS (2016). doi:10.5220/0006034600210032

3. Marshman, E., Van Bolderen, P.M. Interlinguistic variation and lexical knowledge patterns. In: Madsen B.N, Thomsen H.E, (eds.) Managing ontologies and lexical resources: Internationale Sprogstudier og Vidensteknologi, pp. 263-278, Litera, Copenhagen, (2012)

4. Sabol, V.: Visual Analysis of Relatedness in Dynamically Changing Repositories. In: MOVEREALthematic school, Fréjus (2016)

5. Blaise, J.Y., Dudek, I.: Analyzing Alternative Scenarios of Evolution in Heritage Architecture: Modelling and Visualization Challenges. In: Journal of Multimedia Processing and Technologies, pp. 29-48., Vol. 3, no. 1, (2012)

6. Blaise, J.Y., Dudek, I.: Spotting temporal co-occurrence patterns: the historySkyline visual metaphor. In: Proceedings of the 1st International Conference on Metrology for Archaeology, pp. 378-383, Benevento, ISBN: 978-88-940453-3-8, (2015) 
7. Matthew Paris Itinerary to the Holy Land (ca. 1250 A.D.), http://cartographic-images.net/Cartographic_Images/225.1_Palestine.html visited 01-03-2017

8. Rosenberg, D., Grafton, A.: Cartographies of Time: A History of the Timeline. Architectural Press, Princeton (2012)

9. Yabuuchi, A.: Home to School diagram. In: Informational Diagram Collection, p.215, Pie Books, Tokyo (2009)

10. Oelke, D.: Visual document analysis: Towards a semantic analysis of large document collections. PhD dissertation, University of Konstanz, (2010)

11. Marazzato, R., Sparavigna, A.C.: Extracting Networks of Characters and Places from Written Works with CHAPLIN, CoRR - Computing Research Repository, https://arxiv.org/abs/1402.4259 (2014)

12. Bilenko, N.Y., Miyakawa, A.: Visualization of Narrative Structure Analysis of sentiments and character interaction in fiction. http://vis.berkeley.edu/courses/cs294-10-fa13/wiki/images/7/7b/AMNBpaper.pdf (2013)

13. Marshman, E., L'Homme, M.C., Surtees, V.: Verbal markers of cause-effect relations across corpora. In: Madsen B.N, Thomsen H.E, (eds.) Managing ontologies and lexical resources,: Internationale Sprogstudier og Vidensteknologi, pp. 159-174, Litera. Copenhagen (2008)

14. Spence, R.: Information Visualization. Pearson Addison-Wesley ACM Press, Harlow (2001)

15. Thomas, J.J., Cook, K.A.: Illuminating the Path: The Research and Development Agenda for Visual Analytics. IEEE Comput. Graph. Appl. 26, 1, pp. 10-13 (2006)

16. Oelke, D., Spretke, D.; Stoffel, A.; Keim, D.:Visual readability analysis: How to make your writings easier to read. In: IEEE Symposium on Visual Analytics Science and Technology, pp.123-130,VAST, (2010)

17. Koch, S., John, M., Wörner, M., Müller, A., Ertl T.:VarifocalReader - In-Depth Visual Analysis of Large Text Documents. In: IEEE Transactions on Visualisation and computer graphics pp. 1723-1732, Vol2. N.12 (2014)

18. Vuillemot, R., Clement, T., Plaisant, C., Kumar, A.: What's being said near 'Martha'? Exploring name entities in literary text collections. In: IEEE Symposium on Visual Analytics Science and Technology, pp. 107-114, VAST (2009)

19. Wanner, F., Fuchs, J., Oelke, D., Keim, D.A.,: Are my Children Old Enough to Read these Books? Age Suitability Analysis. In: Polibits: research journal on computer science and computer engineering with applications, 43, pp. 93-100 (2011)

20. Kergosien, E., Laval, B., Roche, M., Teisseire, M. : Are opinions expressed in land-use planning documents?. In: International Journal of Geographical Information Science. vol. 28, issue 4, pp. 739-762 (2014)

21. Blaise, J.Y., Dudek, I.: Using abstraction levels in the visual exploitation of a knowledge acquisition process. In: Proceedings of I-Know 2005, pp. 543-552, Graz (2005)

22. Blaise, J.Y., Dudek, I,: Profiling artefact changes: a methodological proposal for the classification and visualisation of architectural transformations. In: Digital Heritage, Proceedings of VSMM 2008 - Virtual Systems and Multimedia, pp. 349-356, Archeolingua, Budapest (2008)

23. Aigner, W., Miksch, S., Schumann, H., Tominski, C.: Visualization of Time-Oriented Data. Human-Computer Interaction Series, Springer-Verlag, London (2011)

24. Sabol, V.: Visualisation in the Web. http://kti.tugraz.at/sta$\mathrm{ff} /$ vsabol/courses/mmis1/slides_vis.pdf

25. Korzybski, A.: The role of language in the perceptual processes. In: Blake, R., Ramsey, G., (eds) Perception: An Approach To Personality, The Ronald Press Company, New York, pp. 170-205 (1951)

26. Tufte, E.R.: Envisioning information. Graphics Press, Cheshire (2001) 